\title{
Compact and Low Loss Microwave Idlers for Low Frequency Integrated Circuits
}

\author{
Shailendra Singh ${ }^{1}$, Subhash Chandra Bera ${ }^{1}$, Dhaval Pujara ${ }^{2}$ \\ ${ }^{1}$ Space Applications Centre, Indian Space Research Organization, Ahmedabad, India \\ ${ }^{2}$ Institute of Technology, Nirma University, Ahmedabad, India \\ *corresponding author, E-mail: contact.singh@yahoo.com
}

\begin{abstract}
Two design methodologies for realization of low frequency (less than $20 \mathrm{GHz}$ ) compact and low loss microwave idlers have been proposed in this paper. Such idlers can be used for realizing low frequency higher order (6X or more) harmonic mixers or multipliers on monolithic integrated technology. Low frequency higher order harmonic mixers or multipliers are generally avoided due to higher losses and board space consumed by multiple idlers. The present proposed methods of idler design are based on realization of idlers by combining distributed microstrip transmission line and lumped components. The approach helps in transmitting the desired frequency with lower insertion loss and providing more rejection to the undesired frequencies. The design proposal has been demonstrated by designing an idler for $3 \mathrm{GHz}$ local oscillator (LO) side of a $6 \mathrm{X}$ harmonic monolithic microwave integrated circuit (MMIC) mixer. This mixer utilizes 6th harmonic of the $3 \mathrm{GHz} \mathrm{LO}$ for generating $18 \mathrm{GHz}$ output radio frequency (RF) signal by frequency mixing. The idler for $3 \mathrm{GHz} \mathrm{LO}$ rejects direct current (dc), intermediate frequency (IF) and selective even harmonics of LO; $6 \mathrm{GHz}, 12 \mathrm{GHz}$ and $18 \mathrm{GHz}$. On wafer test results of the developed 6X harmonic MMIC mixer has substantiated the idler design.
\end{abstract}

\section{Introduction}

Microwave idlers are integral parts of higher order harmonic multipliers and mixers. These are regularly used to selectively suppress unwanted harmonics in resonant terminations to elevate the desired harmonic component and thus improve conversion efficiency. The number of idlers to be used in a circuit depends on the order of mixing or multiplication. The higher the order of mixing or multiplication, the more are the number of idlers used and larger is the footprint area or board space of the circuits.

Idlers are realized using distributed transmission lines or lumped components. Distributed transmission lines require more board space but have lesser losses, whereas, lumped component idlers are compact, but possess higher insertion losses [1]. This issue of space availability versus losses is a major road block in the design of higher order harmonic frequency translation modules [1-3]; which in turn is the reason that $2 \mathrm{X}$ harmonic mixer integrated circuits (ICs) are most popular followed by only few instances of realization of $3 \mathrm{X}$ and $4 \mathrm{X}$ mixers [4-11]. The $2 \mathrm{X}$ mixers referred in [4-7] have to utilize only a single resonator or idler and consume lesser board space. Hence, these have been realized as distributed transmission lines to achieve good rejection and lower losses.

At high frequency of operation, typically at millimeterwave, multiple idlers are feasible as these can be realized using microstrip distributed transmission lines. The 36 $\mathrm{GHz}, 60 \mathrm{GHz}, \mathrm{W}$-band and $320 \mathrm{GHz} 4 \mathrm{X}$ mixers of [9-12] use periodicity of the distributed transmission line of a single idler to reject $2 \mathrm{X}$ and $4 \mathrm{X}$ of the $\mathrm{LO}$ frequency. At higher frequencies, distributed transmission lines have smaller wavelengths, thus consuming lesser chip area and inherently possessing lesser losses.

At frequencies lesser than $20 \mathrm{GHz}$, realization of passive components (like idlers) is usually carried out using lumped R-L-C elements for achieving compactness. This leads to an increase in insertion loss at desired frequency and degradation of rejection at idler frequencies [13-14]. Optimization techniques for idler design [15-16] do not help due to the inherent losses of lumped components.

In this work, two improvement techniques for realizing low loss and higher rejection idlers based on lumped component approach have been proposed and demonstrated using MMIC foundry design kit for a specific case. The idler was used in higher order sub-harmonic I-Q mixer, the on-wafer test result for which has been presented. In spite of idlers being major contributors for conversion efficiency of frequency conversion units, dedicated efforts towards idler design is a less touched upon topic in literature and there are no benchmarks in the said field. Hence, to carry out comparison, the results of the designed multi-frequency idler have been compared with microstrip line based idler design.

The design demonstrated in this paper is an idler designed for LO section of a 6th harmonic (6X) I-Q mixer which uses 6th harmonic $(18 \mathrm{GHz})$ of $3 \mathrm{GHz}$ LO for frequency mixing. The function of this $\mathrm{LO}$ section idler is to let propagate the $3 \mathrm{GHz} \mathrm{LO}$ frequency with minimum insertion loss and to reject dc, IF (100 MHz) and selective even harmonics of LO; $6 \mathrm{GHz}, 12 \mathrm{GHz}$ and $18 \mathrm{GHz}(2 \mathrm{X}$, $4 \mathrm{X}$ and $6 \mathrm{X}$ ). The design has been carried out using the 
PH25 MMIC process design kit (PDK) of the France foundry, United Monolithic Semiconductors (UMS) and using the software, Advanced Design System (ADS). Figure 1 shows block diagram of the 6th harmonic (6X) mixer.

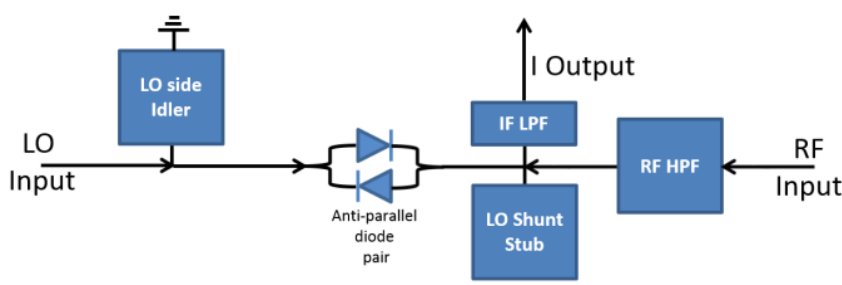

Figure 1: Block diagram of the $6 \mathrm{X}$ mixer.

\section{Design Methodology}

The mixer of figure 1 uses an anti-parallel diode pair for achieving harmonic mixing. For attaining best conversion loss, the IF, RF (6X) and other harmonics of LO i.e. $2 \mathrm{X}$ and $4 \mathrm{X}$ should be rejected.

Lumped component based idlers are realized by the classical series L-C resonator whose resonating frequency is defined by (1):

$$
f_{r}=1 /(2 \pi \sqrt{L C})
$$

Where fr is resonant frequency (Hertz), L is inductance (Henry) and C is capacitance (Farad).

Since lumped components are non-periodic in nature, each frequency to be rejected by the idler would require a different series L-C resonator. Using (1), an idler for suppressing $6 \mathrm{GHz}(2 * \mathrm{LO})$ frequency was designed with the UMS foundry kit. Its layout and simulation results are shown in figure 2 . The idler which rejects one single frequency, $6 \mathrm{GHz}$, occupies $0.15 \mathrm{~mm} \times 0.4 \mathrm{~mm}$ of area. The rejection achieved at $6 \mathrm{GHz}$ is lesser than $20 \mathrm{~dB}(18 \mathrm{~dB}$ here); the insertion loss and return loss at the desired frequency of $3 \mathrm{GHz}$ is $0.7 \mathrm{~dB}$ and $8.9 \mathrm{~dB}$ respectively.

\subsection{Improvement-1: Idler realization by hybrid approach of microstrip line and L-C Lumped component}

A high impedance short length transmission line having electrical length ' $\theta$ ' lesser than 45 degree can be approximated as an inductor [14, 17] with its inductive reactance ' $\mathrm{XL}$ ' defined by (2):

$$
X_{L}=w * L_{t}=Z_{\text {high }} * \sin \theta
$$

Where ' $\mathrm{Lt}$ ' is the inductance obtained from the ' $\theta$ ' length of the transmission line.

$$
L_{t}=\left(Z_{h i g h} * \sin \theta\right) /(2 * \pi * f)
$$
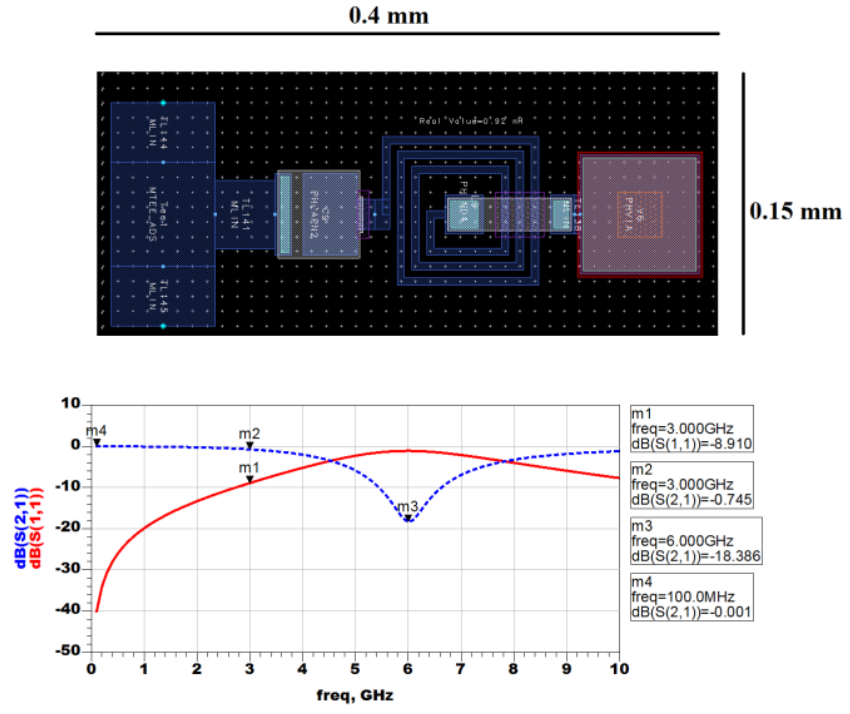

Figure 2: Layout of optimized L-C resonator idler at $6 \mathrm{GHz}$ and its simulated S-parameter response.

Unlike the lumped inductor, value of inductance ' $\mathrm{Lt}$ ' is dependent on the frequency propagating through the line and electrical length of the line at that frequency. As distributed transmission lines fare lesser resistive losses than lumped components [13], using a short length of transmission line in series with L-C lumped component resonator would retain the low loss properties of transmission lines as well as the compactness of the lumped components. Designers would have a choice of compactness along with better rejection and lesser insertion loss. Figure 3 shows a schematic of such a network. Since, the transmission line is equivalent to lumped inductor ' $\mathrm{Lt}$ ' in series to L-C (figure 3), effectively it adds to the value of inductance 'Ls' making it 'Ls $+\mathrm{Lt}$ '. Hence (1) can be rewritten as:

$$
f_{r}=1 /\left(2 \pi \sqrt{\left(L_{s}+L_{t}\right) * C_{s}}\right)
$$

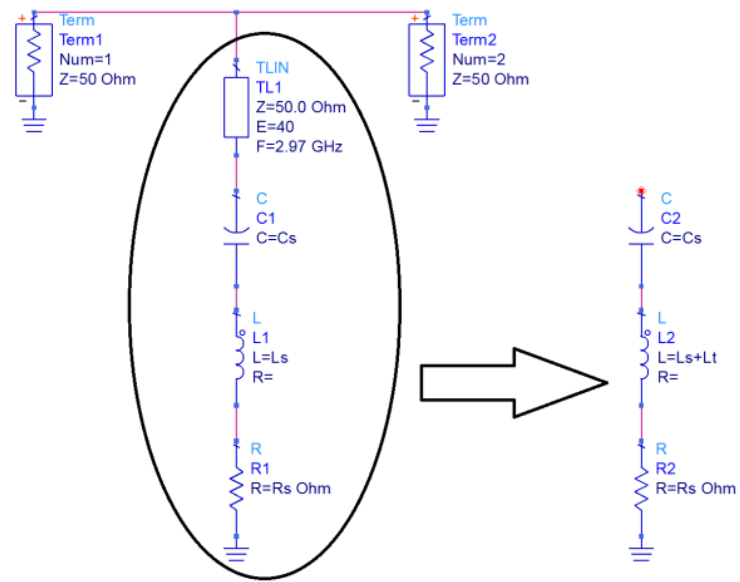

Figure 3: Schematic of hybrid idler approach consisting of both microstrip line and lumped L-C elements. 
Therefore, three parameters, namely, 'Ls', 'Lt' and 'Cs' determine the resonant frequency ' $\mathrm{fr}$ '. It should be noted that the value of ' $\mathrm{Lt}$ ' is for ' $\mathrm{fr}$ ' frequency making it

$f_{r}=1 /\left(2 \pi \sqrt{\left(L_{s}+\left(Z_{\text {high }} * \sin \theta\right) /\left(2 * \pi * f_{r}\right)\right) * C_{s}}\right)$

' $\theta$ ' is chosen such that after synthesis the actual line length is compact as per the designer's needs. This gives the value of inductance contribution ' $\mathrm{Lt}$ ' of the transmission line from (3). Next, an arbitrary value of ' $\mathrm{C}$ ' is selected, from which, the lumped component 'Ls' can be found by extending (1):

$$
L_{s}=\left(1000 /\left(C *\left(2 \pi f_{r}\right)^{\wedge} 2\right)-L_{t}\right.
$$

Such a network has been synthesized and optimized assuming a 25 degree electrical length of the microstrip transmission line at $6 \mathrm{GHz}$. Figure 4 shows the schematic of the network and L-C values obtained from (3) and (6). Figure 5 shows the simulated S-parameter response of the network. It may be seen that there is remarkable improvement in the rejection at $6 \mathrm{GHz}$, from $18 \mathrm{~dB}$ (figure 2) to $27 \mathrm{~dB}$ (figure 5). But, the insertion loss at $3 \mathrm{GHz}$ is still worse.

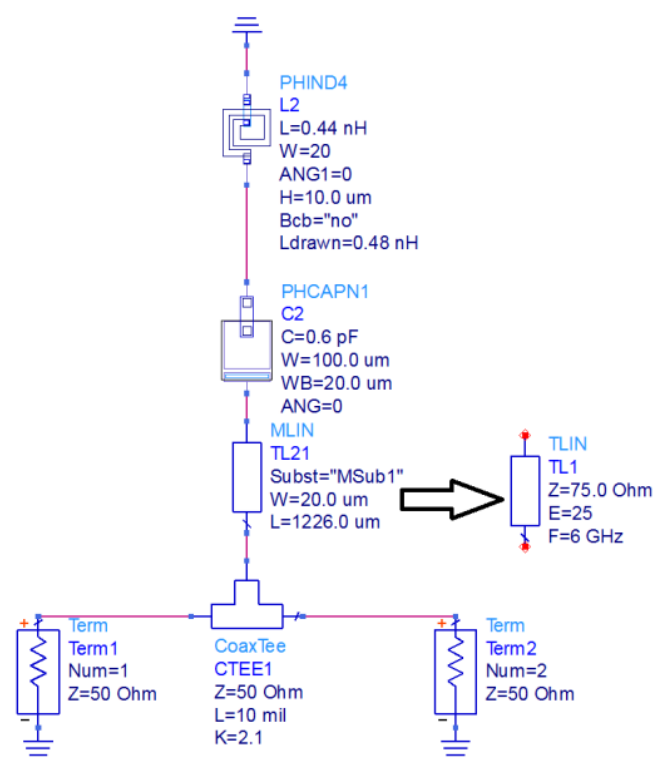

Figure 4: Schematic of optimized $6 \mathrm{GHz}$ hybrid idler.

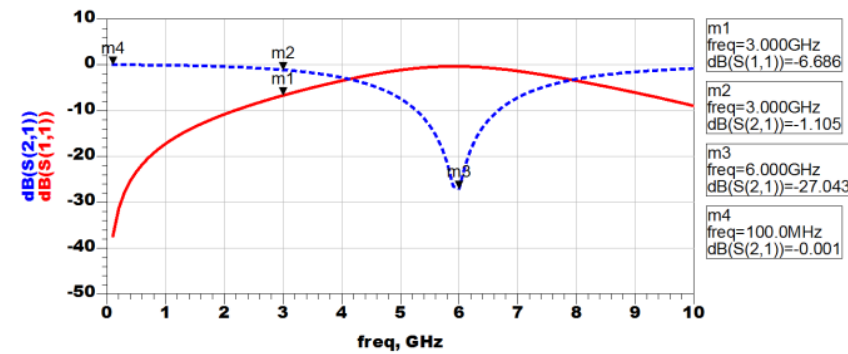

Figure 5: Simulated S-parameter response of the optimized $6 \mathrm{GHz}$ hybrid idler.

\subsection{Improvement-2: Improvement using parallel} inductor

Although the series transmission line L-C hybrid network discussed above improves the rejection at $6 \mathrm{GHz}(2 \mathrm{X})$, it suffers from the following two shortcomings:

(1) The network offers no rejection to dc or IF frequencies. So a separate idler would be required for rejecting dc or IF.

(2) The network suffers from high insertion loss (1.1 $\mathrm{dB})$ at the desired LO frequency $(3 \mathrm{GHz}$ in this case).

Adding an inductor in parallel with the L-C network as shown in figure 6 eliminates the two shortcomings. Analyzing the impedance presented by the network of figure 6 in terms of the inductive reactance ' $\mathrm{XL}$ ' and capacitive reactance ' $\mathrm{XC}$ '; the net impedance offered by the network is:

$Z=\left[\left(X_{C s}+X_{L S}\right) * X_{L p}\right] /\left(X_{C S}+X_{L S}+X_{L p}\right.$

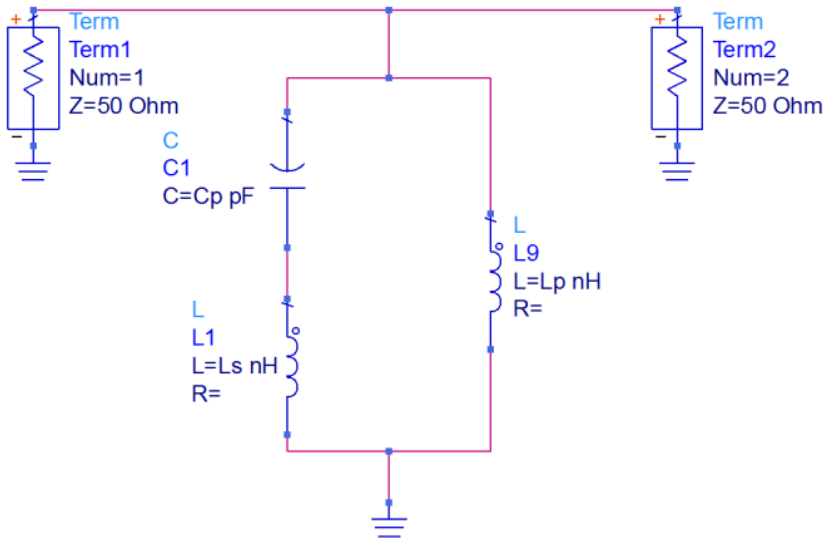

Figure 6: Improved series L-C idler with parallel inductor.

From (7), it can be observed that the impedance offers a short circuit to the following resonant frequencies fr 1 and fr2 rejecting these:

$$
\begin{gathered}
f_{r 1}=1 /\left(2 \pi \sqrt{L_{s} * C_{s}}\right) \\
f_{r 2}=0
\end{gathered}
$$

Where ' $\mathrm{fr} 1$ ' is the L-C series resonant frequency of (1). fr $2=0$ rejects dc signal and offers significant rejection to all IF frequencies near dc. The third resonant frequency is the most interesting outcome of this network.

$$
f_{r 3}=1 /\left(2 \pi \sqrt{\left(L_{s}+L_{p}\right) * C_{s}}\right)
$$

At frequency ' $f r 3$ ', according to (7), the network shows open impedance (infinite impedance). (10) can be used to 
select the value of 'Lp' such that the network shows an open impedance to the desired LO frequency.

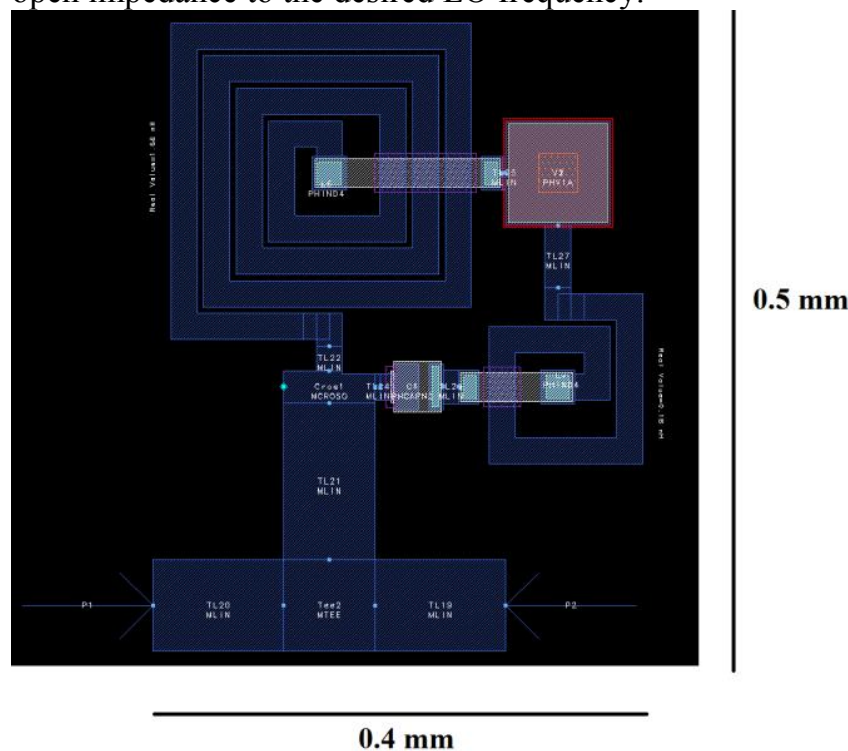

Figure 7: Layout of improved and optimized series L-C idler with parallel inductor.

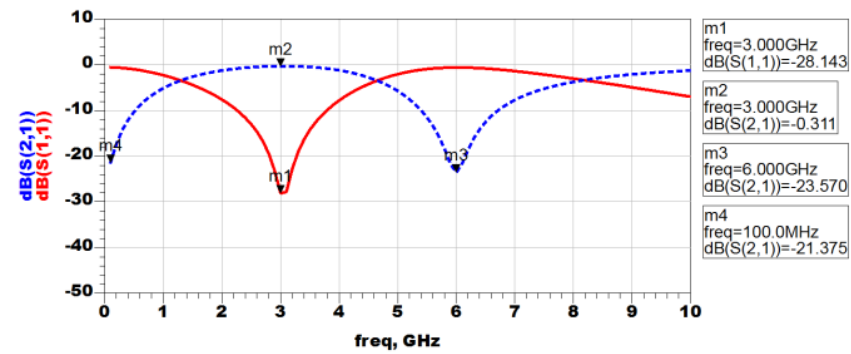

Figure 8: S-parameter simulated response of improved series L-C idler with parallel inductor.

Table 1. Comparison of the original series L-C resonators with the two proposed improvements

\begin{tabular}{|c|c|c|c|}
\hline Parameter & $\begin{array}{l}\text { Series L-C } \\
\text { resonator }\end{array}$ & $\begin{array}{l}\text { Series } \\
\text { transmission } \\
\text { line L-C } \\
\text { hybrid idler }\end{array}$ & $\begin{array}{l}\text { Series } \mathrm{L}-\mathrm{C} \\
\text { resonator } \\
\text { with parallel } \\
\mathrm{L}\end{array}$ \\
\hline Insertion loss at $3 \mathrm{GHz}$ & $0.75 \mathrm{~dB}$ & $1.1 \mathrm{~dB}$ & $0.3 \mathrm{~dB}$ \\
\hline Return loss at $3 \mathrm{GHz}$ & $8.9 \mathrm{~dB}$ & $7 \mathrm{~dB}$ & $28 \mathrm{~dB}$ \\
\hline $\begin{array}{l}\text { Rejection at } 100 \mathrm{MHz} \\
\text { (IF) }\end{array}$ & $0 \mathrm{~dB}$ & $0 \mathrm{~dB}$ & $21 \mathrm{~dB}$ \\
\hline Rejection at $6 \mathrm{GHz}(2 \mathrm{X})$ & $18 \mathrm{~dB}$ & $27 \mathrm{~dB}$ & $23 \mathrm{~dB}$ \\
\hline
\end{tabular}

Using these synthesis formulae, the layout in figure 7 was built for including the shunt Inductor ' $L p$ ' to get minimum insertion loss at $3 \mathrm{GHz}$ LO frequency. Figure 8 shows its simulated S-parameter response. It may be observed that the idler network has become more frequency selective, with significant improvement in return loss $(28$ $\mathrm{dB})$ and insertion loss $(0.32 \mathrm{~dB})$ at LO frequency and good rejection at $2 * \mathrm{LO}$ frequency $(23 \mathrm{~dB})$. In addition, the network offers considerable rejection $(21 \mathrm{~dB})$ to $100 \mathrm{MHz}$
IF frequency. These findings have been tabulated in table 1 for comparison of the two improvements with the series L$\mathrm{C}$ resonator.

\section{Development of the improved idler and associated mixer}

To realize multi-frequency idler at $2 * \mathrm{LO}, 4 * \mathrm{LO}$ and $6 * \mathrm{LO}$, three L-C series resonant networks would be required along with the parallel inductor for rejecting $\mathrm{dc}$ and IF and ensuring acceptable insertion loss at LO. Using the hybrid microstrip line and lumped component approach described in this paper, a network was synthesized for rejecting dc, $100 \mathrm{MHz}$ IF, $6 \mathrm{GHz} 2 * \mathrm{LO}, 12 \mathrm{GHz} 4 * \mathrm{LO}$ and $18 \mathrm{GHz}$ 6* LO signals and passing $3 \mathrm{GHz}$ LO signal with minimum attenuation. The layout of the network is shown in figure 9 and its s-parameter simulation results are shown in figure 10 . As seen, this network consists of four resonators with additional optimized length of transmission lines to decrease the losses. The 4 resonators act as individual idlers to suppress dc/IF, $6 \mathrm{GHz}, 12 \mathrm{GHz}$ and $18 \mathrm{GHz}$ signal. The dc idler has been designed in conjunction with (10) to offer minimum attenuation to $3 \mathrm{GHz}$. The size of the layout is approximately $0.5 \mathrm{~mm} \times 0.75 \mathrm{~mm}$.

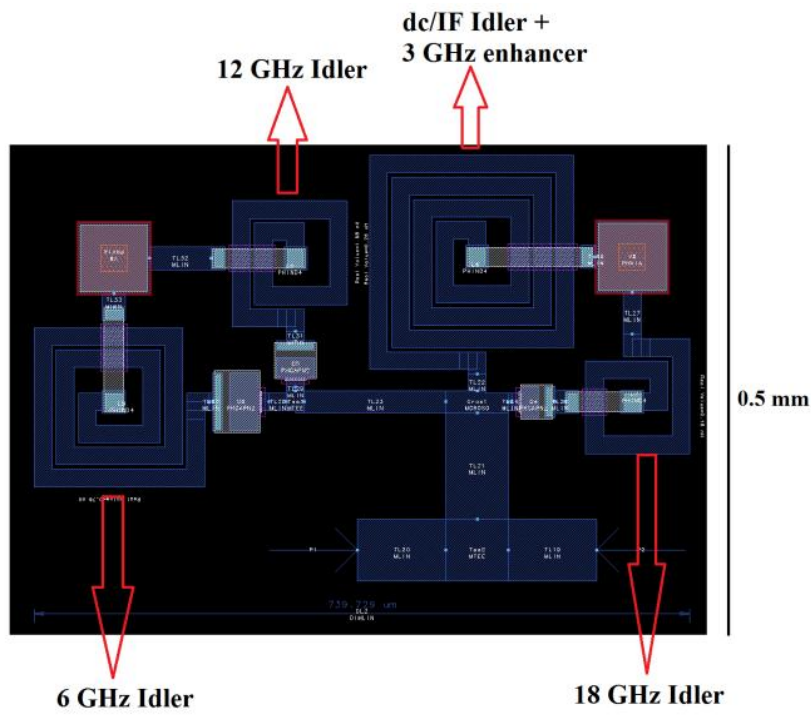

$0.75 \mathrm{~mm}$

Figure 9. Optimized layout of the multi-frequency idler.

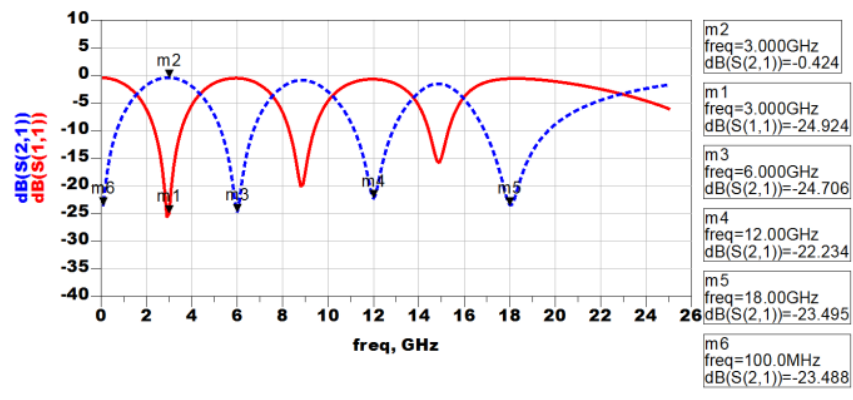

Figure 10: S-parameter simulation of the multi-frequency idler. 
As no benchmark exists for the comparison of idlers, a microstrip line shunt stub idler with quarter-wavelength at LO frequency (Figure 11) was designed and optimized. The size of the idler is $1.2 \mathrm{~mm} \times 1.7 \mathrm{~mm}$. Table 2 shows a comparison between the two realization approaches. The proposed idler occupies almost one-fifth lesser board area when compared to the microstrip line idler layout. The improved lumped component idler was used to realize the 6X I-Q Mixer. On-wafer measured up-conversion loss (Figure 12) of the $6 \mathrm{X}$ mixer was $15.5 \mathrm{~dB}$ with $29 \mathrm{~dB}$ of side band rejection and $62 \mathrm{~dB}$ LO to RF isolation. Size of the chip is $3.2 \mathrm{~mm} \times 2.6 \mathrm{~mm}$. This conversion efficiency and compact size was possible only due to the compact idler design.

Table 2. Comparison of the proposed improved lumped component based idler with microstrip line based idler

\begin{tabular}{|c|c|c|}
\hline Parameter & Microstrip line Idler & $\begin{array}{l}\text { Improved lumped } \\
\text { component idler }\end{array}$ \\
\hline Insertion loss at $3 \mathrm{GHz}$ & $0.1 \mathrm{~dB}$ & $0.4 \mathrm{~dB}$ \\
\hline Return loss at $3 \mathrm{GHz}$ & $42 \mathrm{~dB}$ & $24 \mathrm{~dB}$ \\
\hline $\begin{array}{l}\text { Rejection at } 100 \mathrm{MHz} \\
\text { (IF) }\end{array}$ & $23 \mathrm{~dB}$ & $19 \mathrm{~dB}$ \\
\hline Rejection at $6 \mathrm{GHz}(2 \mathrm{X})$ & $27 \mathrm{~dB}$ & $24 \mathrm{~dB}$ \\
\hline Rejection at $12 \mathrm{GHz}(4 \mathrm{X})$ & $23 \mathrm{~dB}$ & $22 \mathrm{~dB}$ \\
\hline Rejection at $18 \mathrm{GHz}(6 \mathrm{X})$ & $21 \mathrm{~dB}$ & $23 \mathrm{~dB}$ \\
\hline Size & $1.2 \mathrm{~mm} \times 1.7 \mathrm{~mm}$ & $\begin{array}{l}0.5 \mathrm{~mm} \times 0.75 \mathrm{~mm} \\
(5 \text { times lesser) }\end{array}$ \\
\hline
\end{tabular}
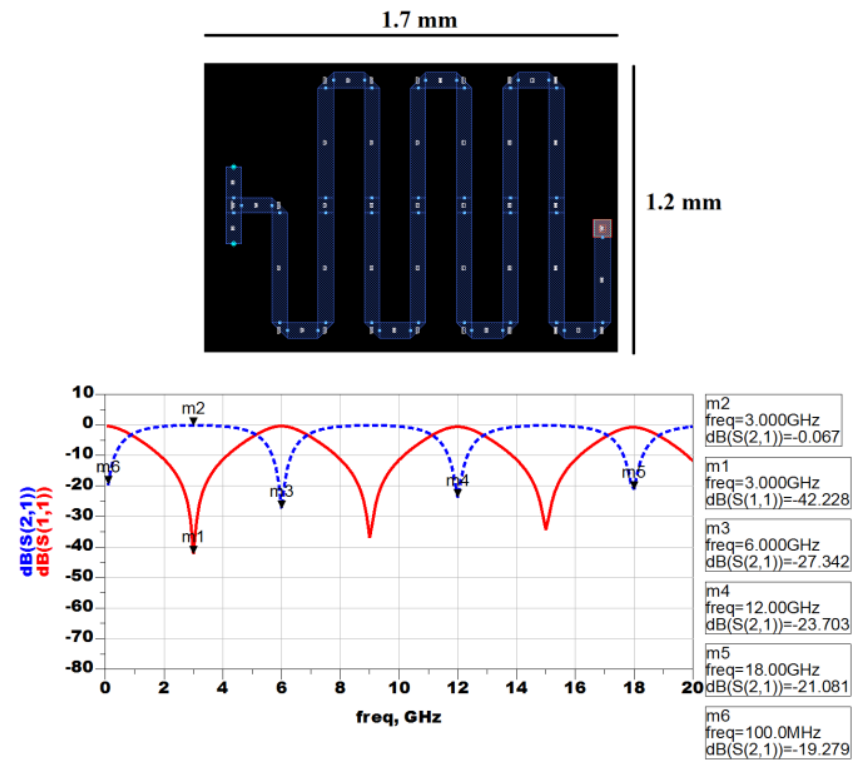

Figure 11. Layout of optimized microstrip line quarter-wave length short stub at $3 \mathrm{GHz}$ and its simulated S-parameter response.

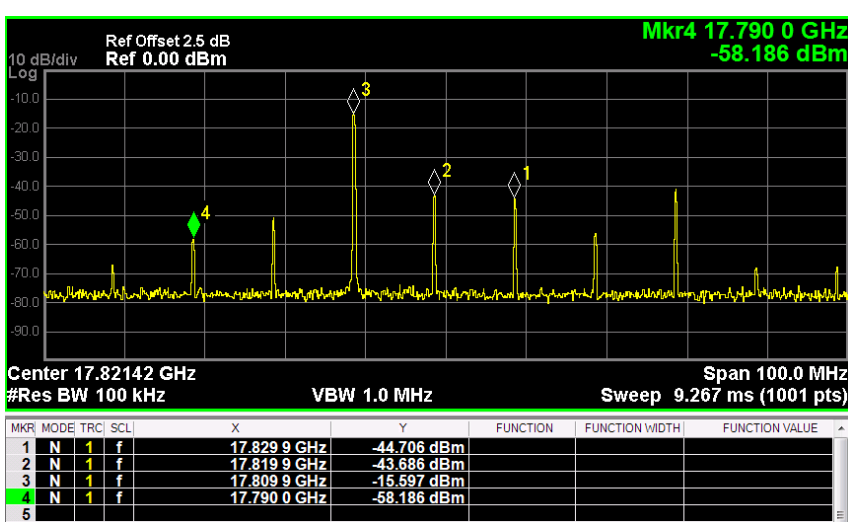

Figure 12. On-wafer measured results of the $18 \mathrm{GHz} 6 \mathrm{X} \mathrm{I-Q}$ Mixer; LO input is $2.97 \mathrm{GHz}$ at $+19 \mathrm{dBm}, \mathrm{I} / \mathrm{Q}$ input is 10 $\mathrm{MHz}$ at $0 \mathrm{dBm}$.

\section{Conclusions}

In this work, two techniques for realizing low loss and better rejection lumped component idlers have been proposed and demonstrated for $3 \mathrm{GHz} \mathrm{LO}$ frequency idler. Using both the techniques together, a multi-frequency idler was designed. The designed idler shows comparable results with microstrip line based idler that tends to be larger in area at lower frequencies. Due to its compact size, the designed idler can be comfortably utilized for realizing complex RF ICs like I-Q mixers in lesser chip area for much higher harmonic mixing and multiplication; ensuring good conversion efficiency at optimal LO power owing to its lower insertion loss.

\section{References}

[1] S. A. Maas, Nonlinear Microwave and RF Circuits, pg. 360, Norwood, MA, Artech House (2003).

[2] Mike Golio, RF and Microwave Semiconductor Device Handbook, pg. 1-10, Boca Raton, FA, Taylor and Francis (2003).

[3] T.G. Roer, Microwave Electronic Devices, pg. 252-260, India, Springer (1994).

[4] Bo Zhang, Byron Alderman, Zhe Chen, Yong Fan, Xiaofan Yang, and Xiaobo Yang, The Design of a 200240-GHz Sub-Harmonic Mixer Based on RAL's Planar Schottky Diodes. Terahertz Science and Technology, vol. 4, No. 3 (Sept. 2011).

[5] S. Shamsinejad, M. Soleimani, M. Tayarani, and N. Komjani, Novel Even Harmonic Mixer for 3G Mobile Receivers. Progress In Electromagnetics Research M, Vol. 1, 69-77 (2008).

[6] Zhe Chen, Bo Zhang, Yong Fan, Shixi Zhang, and Xiaofan Yang, Design of a 118-GHz Sub-Harmonic Mixer Using Foundry Diodes, Proceedings of International Symposium on Signals, Systems and Electronics (2010).

[7] Kaixue Ma and Jianguo Ma, A $23 \mathrm{GHz}$ High Isolation Sub-Harmonic Mixer, IEEE Explore, (2005).

[8] Jian-An Hou and Yeong-Her Wang, A Ka Band Balanced Third LO-Harmonic Mixer Using a Lumped- 
Elements Quadrature Hybrid, IEEE Microwave and Wireless Components Letters, vol., No. 6 (June 2008).

[9] Michael W. Chapman and Sanjay Raman, A 60-GHz Uniplanar MMIC 4 Subharmonic Mixer, IEEE Transactions On Microwave Theory and Techniques, vol. 50, No. 11 (Nov. 2002).

[10]Bo Xiang, Wenbin Dou, Minmin He, and Zongxin Wang, Research on Fourth Harmonic Mixer at W Band in the Imaging System, Journal of the Korean Institute of Electromagnetic Engineering and Science, vol. 10, No. 4 (Dec. 2010).

[11] Chao Liu, Qiang Li, Yihu Li, Xiang Li, Haitao Liu, and Yong-Zhong Xiong, Design of $340 \mathrm{GHz} 2 \times$ and $4 \times$ Sub-Harmonic Mixers Using Schottky Barrier Diodes in Silicon-Based Technology, Micromachines (2015), 6, 592-599; doi:10.3390/mi6050592.

[12] Quan Xue, Member, Kam Man Shum, Member, and Chi Hou Chan, Low Conversion-Loss Fourth Subharmonic Mixers Incorporating CMRC for Millimeter-Wave Applications, IEEE Transactions on Microwave Theory and Techniques, vol. 51, No. 5 ( May 2003).

[13] Inder Bahl, Lumped Elements for RF and Microwave Circuits, pg. 353-394, Artech House, (2002).

[14] Jia-Shen G. Hong and M.J. Lancaster, Microstrip filters for RF/Microwave Applications, pg. 94, John Wiley \& Sons (2004).

[15] Asher Madjar, A generic approach to optimum design of microwave and millimeter wave subharmonic mixers, 25th European Microwave Conference, (1995), IEEE Xplore. DOI: 10.1109/EUMA.1995.337159

[16] Asher Madjar, Izhak Shappir, and Saul Zoref, Improvement of the Generic Approach to Optimum Design of Microwave and Millimeter Wave Subharmonic Mixers, 27th European Microwave Conference (1997), IEEE Xplore. DOI: 10.1109/EUMA.1997.337869

[17] David M. Pozar, Microwave Engineering, 4th edition, pg. 66, USA, John Wiley \& Sons (2012). 